\title{
A KAM Theorem for Lower Dimensional Elliptic Invariant Tori of Nearly Integrable Symplectic Mappings
}

\author{
Shunjun Jiang \\ College of Sciences, Nanjing Tech University, Nanjing 210009, China \\ Correspondence should be addressed to Shunjun Jiang; jiangshunjun@njtech.edu.cn
}

Received 23 October 2016; Accepted 20 February 2017; Published 16 March 2017

Academic Editor: Hugo Leiva

Copyright ( 2017 Shunjun Jiang. This is an open access article distributed under the Creative Commons Attribution License, which permits unrestricted use, distribution, and reproduction in any medium, provided the original work is properly cited.

This paper develops a new KAM theorem for a class of lower dimensional elliptic invariant tori of nearly integrable symplectic mappings with generating functions but without assuming any nondegenerate condition.

\section{Introduction and Main Results}

The research on nondegeneracy condition of Hamiltonian systems is the fundamental problem of KAM theory because of small divisor problem. There are a significant number of results on nondegeneracy condition. We refer to Kolmogorov [1], Bruno [2], Rüssmann [3, 4], and Xu et al. [5]. The proofs of classic KAM theorems [1, 6-8] are based on the KAM iteration procedure, which include some nondegeneracy conditions. Can the nondegeneracy condition be separated from KAM iteration procedure? In 2016, Xu and $\mathrm{Lu}$ [9] provided a new KAM technique to prove some general KAM theorems for nearly integrable Hamiltonian systems without any nondegeneracy condition. $\mathrm{Xu}$ and $\mathrm{Lu}$ also generalize some classic KAM theorems [1, 6-8] by the general KAM theorems in [9] combining some nondegeneracy conditions. We note that although the proofs of the KAM theorems in [9] did not need nondegeneracy condition, the assumption of nondegeneracy condition is still necessary for the application of those theorems; otherwise they may have no dynamical consequences. We refer to [9] for more details.

As one part of the classical KAM theory, the persistence of invariant tori of nearly integrable twist mappings was investigated by lots of mathematicians. The first work was due to Moser $[7,8]$. Since then, a large body of KAM results for mappings have been proved. We refer to [10-17]. As discrete Hamiltonian systems, symplectic mappings are special among all the mappings because they have symplectic structures, and hence they attract many mathematicians' attention. In 2015, Lu et al. [18] proved a KAM theorem on lower dimensional elliptic invariant tori for nearly integrable symplectic mappings. We refer readers to [19-21] for more results on symplectic mappings.

Motivated by [9, 18, 22], we will extend the result [9] to symplectic mappings and prove a KAM theorem of symplectic mappings with generating functions but without assuming any nondegenerate condition.

We consider the following parameterized symplectic mapping $\Phi(x, u, y, v ; \xi)$ such that

$$
\begin{gathered}
\Phi:(x, u, y, v) \in \mathbb{T}^{n} \times \mathscr{W} \times \mathcal{O} \times \mathscr{W} \longrightarrow \\
(\hat{x}, \widehat{u}, \hat{y}, \widehat{v}) \in \mathbb{T}^{n} \times \mathbb{R}^{m} \times \mathbb{R}^{n} \times \mathbb{R}^{m},
\end{gathered}
$$

and $\xi \in \Pi \subset \mathcal{O}$ is parameter where $\mathcal{O} \subset \mathbb{R}^{n}$ is a bounded closed connected domain and $\Pi$ a subset of $\mathcal{O}$ such that $\operatorname{dist}(\partial \mathcal{O}, \Pi)=\inf _{s \in \Pi, t \in \partial \mathcal{O}}|s-t|>0$. Moreover, $\Phi(x, u, y, v ; \xi)$ is implicitly defined by a generating function

$$
H(x, u, \widehat{y}, \widehat{v} ; \xi)=N+P,
$$

where

$$
\begin{aligned}
N(x, u, \hat{y}, \widehat{v} ; \xi)= & \langle x+\omega(\xi), \hat{y}\rangle+\langle A u, \widehat{v}\rangle \\
& +\frac{1}{2}\langle B u, u\rangle+\frac{1}{2}\langle C \widehat{v}, \widehat{v}\rangle
\end{aligned}
$$

with $A, B$ and $C$ being constant matrices. Moreover, $B$ and $C$ are symmetric. 
There are a great number of results in symplectic mappings and Hamiltonian systems, which are parallel and almost identical, but the proofs are different. The reason lies in special properties of symplectic mappings. For instance, the generating functions, which decide the symplectic mappings and the relation of variables, take on sophisticated implicit forms. So the relation of variables in symplectic mappings is not easily understood, which makes KAM estimates more complicated.

Without assuming any nondegenerate condition, we will give a formal KAM theorem for symplectic mappings. The idea of the proof is to separate the nondegenerate conditions from the KAM iteration, which was introduced in [9]. It is noted that the proofs of the classic KAM theorems [1, 68 ] usually need nondegenerate conditions, which can assure that the small divisor conditions hold at each KAM-step; thus the Diophantine constants $\alpha_{n}$ in the $n$-KAM steps are chosen decreasing and the existence of invariant tori can be guaranteed. However, in this paper, since we do not have any nondegenerate condition, the existence of invariant tori depends on whether the final frequencies $\omega_{*}$ meet the small divisor conditions, where $\omega_{*}=\lim _{n \rightarrow \infty} \omega_{n}$. The key lies in an explicit extension of small divisors to the parameter definition domain and the choice of the Diophantine parameter $\alpha_{n}$. In our KAM iteration we require $\alpha_{n}$ to increase as $n \rightarrow \infty$. Moreover, in our problem there are not only tangential frequency $\omega_{*}$ but also normal frequency $\theta_{*}$; this makes our conditions more completed than in [9]. Although no nondegenerate condition is assumed in our theorem, it is necessary in its application. In fact, we usually use some nondegenerate condition to guarantee the final frequencies $\omega_{*}$ and $\theta_{*}$ to satisfy the nonresonance and so the existence of invariant tori. In particular, the previous KAM results on symplectic mappings can be consequences of our theorem under various kinds of nondegenerate conditions. Without the assumption of nondegenerate conditions, our theorem is only formal and may have no dynamical consequences. tions.

Before giving the main result, we introduce some assump-

Assumption 1 (elliptic condition). Set

$$
\begin{aligned}
& A=\sec \theta, \\
& B=C=\tan \theta,
\end{aligned}
$$

where $\theta=\left(\theta_{1}, \ldots, \theta_{m}\right), \sec \theta=\operatorname{diag}\left(\sec \theta_{1}, \ldots, \sec \theta_{m}\right) \cdot \tan \theta$, $\sin \theta$, and $\cos \theta$ have similar notations.

Remark 2. Consider the symplectic mapping $\Phi(x, u, \widehat{y}, \widehat{v} ; \xi)$ generated by $H(x, u, \widehat{y}, \widehat{v} ; \xi)$ in (2). If $A$ is nonsingular, $\Phi$ can be expressed explicitly as

$$
\begin{aligned}
& \widehat{x}=x+\omega(y), \\
& \widehat{y}=y,
\end{aligned}
$$

$$
\begin{aligned}
& \widehat{u}=\left(A-C\left(A^{T}\right)^{-1} B\right) u+C\left(A^{T}\right)^{-1} v, \\
& \widehat{v}=-\left(A^{T}\right)^{-1} B u+\left(A^{T}\right)^{-1} v .
\end{aligned}
$$

We define

$$
\Omega=\left(\begin{array}{cc}
A-C\left(A^{T}\right)^{-1} B & C\left(A^{T}\right)^{-1} \\
-\left(A^{T}\right)^{-1} B & \left(A^{T}\right)^{-1}
\end{array}\right)_{2 m \times 2 m .}
$$

By Assumption 1, we have

$$
\Omega=\left(\begin{array}{cc}
\cos \theta & \sin \theta \\
-\sin \theta & \cos \theta
\end{array}\right) .
$$

It is easy to verify $\Omega$ has the eigenvalues $e^{ \pm i \theta_{1}}, \ldots, e^{ \pm i \theta_{m}}$. Since $\left|e^{ \pm i \theta_{i}}\right|=1$ and $e^{ \pm i \theta_{1}} \neq 1, i=1,2, \ldots, m$, we call the lower dimensional invariant torus determined by the above symplectic mapping $\Phi$ elliptic.

Assumption 3 (nonresonance conditions). Suppose $\omega$ satisfies the following: for $k \in Z^{n}$ and $1 \leq i, j \leq n$,

$$
\begin{aligned}
&|\langle k, \omega\rangle-2 \pi l| \geq \frac{\alpha}{(1+|k|)^{\tau}}, \\
&\left|\langle k, \omega\rangle-\theta_{j}-2 \pi l\right| \geq \frac{\alpha}{(1+|k|)^{\tau}}, \\
&\left|\langle k, \omega\rangle-\theta_{i}-\theta_{j}-2 \pi l\right| \geq \frac{\alpha}{(1+|k|)^{\tau}}, \\
&\left|\langle k, \omega\rangle+\theta_{i}-\theta_{j}-2 \pi l\right| \geq \frac{\alpha}{(1+|k|)^{\tau}}, \\
&|k|+|i-j| \neq 0 .
\end{aligned}
$$

Set

$$
\begin{aligned}
& \mathscr{T}_{s}=\left\{x \in \frac{\mathbb{C}^{n}}{2 \pi \mathbb{Z}^{n}}:|\operatorname{Im} x|_{\infty} \leq s\right\}, \\
& \mathscr{B}_{r}=\left\{y \in \mathbb{C}^{n}:|y|_{1} \leq r^{2}\right\}, \\
& \mathscr{W}_{r}=\left\{w \in \mathbb{C}^{m}:|w|_{2} \leq r\right\} .
\end{aligned}
$$

Denote $\mathscr{D}(s, r)=\mathscr{T}_{s} \times \mathscr{W}_{r} \times \mathscr{B}_{r} \times \mathscr{W}_{r}$. Here $|x|_{\infty}=\max _{1 \leq j \leq n}\left|x_{j}\right|,|y|_{1}=\sum_{1 \leq j \leq n}\left|y_{j}\right|$ and $|w|_{2}=$ $\left(\sum_{1 \leq j \leq m}\left|w_{j}\right|^{2}\right)^{1 / 2}$. In this paper, we will use the norms defined in [18].

Theorem 4. Consider the parameterized symplectic mapping $\Phi(\cdot ; \xi)$ generated by $H(\cdot ; \xi)$ defined in (2). Let $H$ be real analytic 
in $D(s, r)$ and $C^{m}$-smooth in $\xi$ on $\Pi$. Suppose that Assumptions 1 and 3 hold. Let

$$
\begin{aligned}
O_{\alpha, \tau} & =\left\{(\omega, \theta) \in \mathbb{R}^{n}\right. \\
& \times \mathbb{R}^{m}:\left|\langle k, \omega\rangle-s_{1} \theta_{i}-s_{2} \theta_{j}-2 \pi l\right| \\
& \geq \frac{\alpha}{(1+|k|)^{\tau}},|k|+|i-j| \neq 0, \forall k \in \mathbb{Z}^{n} \backslash\{0\}, \theta \\
& =\left(\theta_{1}, \ldots, \theta_{m}\right),\left|s_{1}\right|+\left|s_{2}\right| \leqslant 2,\left|s_{i}\right| \leqslant 1, s_{i} \in \mathbb{Z}, i \\
& =1,2\} .
\end{aligned}
$$

Then, for any $0<\alpha \leqslant 1, \tau>n-1$ and $m \geqslant 0$, there exists sufficiently small $\epsilon>0$ such that if $\left\|X_{P}\right\|_{r ; \mathscr{D}(s, r) \times \Pi} \leqslant \epsilon$ the following results hold:

(i) There exist a family of symplectic mappings $\left\{\Psi_{*}(\cdot ; \xi)\right.$ : $\xi \in \Pi\}$ with

$$
\left\|\left(\Psi_{*}-i d\right)\right\|_{D(r ; s / 2, r / 2) \times \Pi} \leq c \epsilon,
$$

such that $\Phi_{*}(\cdot ; \xi)=\Psi_{*}^{-1} \circ \Phi \circ \Psi_{*}$ is generated by functions $H_{*}=N_{*}+P_{*}$ as in (2), where

$$
\begin{aligned}
N_{*}(x, u, \hat{y}, \hat{v} ; \xi)= & \left\langle x+\omega_{*}, \hat{y}\right\rangle+\left\langle A_{*} u, \widehat{v}\right\rangle \\
& +\frac{1}{2}\left\langle B_{*} u, u\right\rangle+\frac{1}{2}\left\langle C_{*} \widehat{v}, \widehat{v}\right\rangle, \\
P_{*}(x, u, \hat{y}, \widehat{v} ; \xi)= & \sum_{|i|+|j|+2|| l \mid \geq 3} P_{l i j}(x ; \xi) \hat{y}^{l} u^{i} \widehat{v}^{j} .
\end{aligned}
$$

Let $A_{*}=\sec \theta_{*}$ and $B_{*}=C_{*}=\tan \theta_{*}$. One has

$$
\begin{gathered}
\left\|\omega_{*}-\omega\right\|_{\mathscr{C}^{m}(\Pi)} \leqslant 2 \epsilon, \\
\left\|\theta_{*}-\theta\right\|_{\mathscr{C}^{m}(\Pi)} \leqslant c \epsilon .
\end{gathered}
$$

(ii) If $\left(\omega_{*}(\xi), \theta_{*}(\xi)\right) \in O_{\alpha, \tau}$, the symplectic mapping $\Phi(\cdot ; \xi)$ has an invariant torus $\Psi_{*}\left(\mathbb{T}^{n}, 0 ; \xi\right)$ with frequencies $\omega_{*}(\xi)$.

Remark 5. By Theorem 4, for all $\xi \in\left\{\xi \in \Pi:\left(\omega_{*}(\xi), \theta_{*}(\xi)\right) \in\right.$ $\left.O_{\alpha, \tau}\right\}$, the original symplectic mapping $\Phi(\cdot ; \xi)$ is transformed into the conjugate mappings $\Phi_{*}(\cdot ; \xi)$ under the compatible transformation $\Psi_{*}(\cdot ; \xi)$. Hence the mapping $\Phi(\cdot ; \xi)$ has an invariant torus with the frequency $\omega_{*}(\xi)$. If $\{\xi \in \Pi$ : $\left.\left(\omega_{*}(\xi), \theta_{*}(\xi)\right) \in O_{\alpha, \tau}\right\}=\emptyset$, the conjugate mapping $\Phi_{*}(\cdot ; \xi)$ has nothing to do with the symplectic mapping $\Phi(\cdot ; \xi)$. Then our result is with no sense. To avoid this situation, some nondegeneracy conditions are necessary for $\Phi(\cdot ; \xi)$ to satisfy with $\left\{\xi \in \Pi:\left(\omega_{*}(\xi), \theta_{*}(\xi)\right) \in O_{\alpha, \tau}\right\} \neq \emptyset$. But Theorem 4 itself does not need any nondegeneracy condition.

\section{Proof of Main Results}

2.1. KAM-Step

Lemma 6 (KAM iteration lemma). Consider the symplectic mapping $\Phi(\cdot ; \xi)$ defined in Theorem 4. Let $\alpha \leqslant \alpha_{*} \leqslant$
$2 \alpha, \tau>n-1, m \geqslant 0$. Assume $\omega \in \mathscr{C}^{m}(\Pi)$ and $P$ satisfies $\left\|X_{P}\right\|_{r ; D(s, r) \times \Pi} \leq \epsilon=\eta^{2} \alpha^{2 \bar{v}} \rho^{2 \nu} E$, where $0<\alpha<1,0<\rho<$ $s / 5,0<\eta<1 / 8, \eta^{2} e^{-K \rho}=E$ with $\bar{v}=4(m+1), v=$ $4 \tau(m+1)+m+n$. Then the following conclusions hold:

(i) $\forall \xi \in \Pi$ there exists a symplectic diffeomorphism $\Psi(\cdot ; \xi)$ with

$$
\begin{gathered}
\|\Psi-i d\|_{r ; D(s-3 \rho, r / 4) \times \Pi} \leq \frac{c \epsilon}{\alpha^{\bar{v}} \rho^{\nu}}, \\
\|D \Psi-I d\|_{r ; D(s-3 \rho, r / 4) \times \Pi} \leq \frac{c \epsilon}{\alpha^{\bar{v}} \rho^{\nu+1}},
\end{gathered}
$$

such that conjugate mapping $\Phi_{+}(\cdot ; \xi)=\Psi^{-1} \circ \Phi \circ \Psi$ is generated by $H_{+}=N_{+}+P_{+}$, where

$$
\begin{aligned}
N_{+}= & \left\langle x+\omega_{+}(\xi), \hat{y}\right\rangle+\left\langle A_{+} u, \widehat{v}\right\rangle+\frac{1}{2}\left\langle B_{+} u, u\right\rangle \\
& +\frac{1}{2}\left\langle C_{+} \widehat{v}, \widehat{v}\right\rangle
\end{aligned}
$$

with $A_{+}=\sec \theta_{+}, B_{+}=C_{+}=\tan \theta_{+}$. Moreover, one has

$$
\begin{aligned}
\left\|\omega_{+}-\omega\right\|_{\mathscr{C}^{m}(\Pi)} & \leq \epsilon, \\
\left\|\theta_{+}-\theta\right\|_{\mathscr{C}^{m}(\Pi)} & \leq c \epsilon .
\end{aligned}
$$

$P_{+}$is a smaller term with the estimate

$$
\left\|X_{P}\right\|_{r_{+} ; D\left(s_{+}, r_{+}\right) \times \Pi} \leq c \epsilon_{+}=c \eta_{+}^{2} \alpha_{+}^{2 \bar{v}} \rho_{+}^{2 v} E_{+} .
$$

Here

$$
\begin{aligned}
s_{+} & =s-5 \rho, \\
\rho_{+} & =\sigma \rho, \\
\eta_{+} & =E_{+}, \\
r_{+} & =\eta r, \\
E_{+} & =E^{4 / 3}, \\
\alpha & \leqslant \alpha_{+} \leqslant 2 \alpha .
\end{aligned}
$$

(ii) Let $\alpha_{+}=\alpha+3 K^{\tau+1} \epsilon$ and

$$
\begin{aligned}
O_{\alpha, K} & =\left\{(\omega(\xi), \theta(\xi)) \in \mathbb{R}^{n}\right. \\
\quad & \mathbb{R}^{m}:\left|\langle\omega, k\rangle-s_{1} \theta_{i}-s_{2} \theta_{j}-2 \pi l\right| \\
& \geqslant \frac{\alpha}{(1+|k|)^{\tau}}, \forall k \in Z^{n}, 0<|k| \leq K,\left|s_{1}\right|+\left|s_{2}\right| \\
& \left.\leqslant 2,\left|s_{i}\right| \leqslant 1, \quad s_{i} \in \mathbb{Z}, \quad i=1,2\right\} .
\end{aligned}
$$

Define

$$
\begin{aligned}
\mathscr{U} & =\left\{\xi \in \Pi:(\omega(\xi), \theta(\xi)) \in O_{\alpha, K}\right\}, \\
\mathscr{U}_{+} & =\left\{\xi \in \Pi:\left(\omega_{+}(\xi), \theta_{+}(\xi)\right) \in O_{\alpha_{+}, K_{+}}\right\},
\end{aligned}
$$

where $K_{+}>K$ satisfies $e^{-K_{+} \rho_{+}} / \eta_{+}^{2}=E_{+}$. Then one has $\mathscr{U}_{+} \subset \mathcal{U}$. 
2.1.1. Generating Functions of Conjugate Mappings. For the convenience, let $p=(x, u)$ and $q=(y, v) . \widehat{p}$ and $\hat{q}$ have similar meaning. The symplectic structure becomes $d p \wedge d q$ on $\mathbb{R}^{n+m} \times \mathbb{R}^{n+m}$. Consider a symplectic mapping $\Phi:(p, q) \rightarrow$ $(\widehat{p}, \widehat{q})$ generated by

$$
\begin{gathered}
\widehat{p}=\partial_{\widehat{q}} H(p, \widehat{q}), \\
q=\partial_{p} H(p, \widehat{q}) .
\end{gathered}
$$

The generating function is $H(p, \widehat{q})=N(p, \widehat{q})+P(p, \widehat{q})$, where $N$ is main term and $P$ is a small perturbation.

We need a symplectic transformation $\psi_{1}:\left(p_{+}, q_{+}\right) \rightarrow$ $(p, q)$, generated by

$$
\begin{gathered}
q=q_{+}+F_{1}\left(p, q_{+}\right), \\
p_{+}=p+F_{2}\left(p, q_{+}\right) .
\end{gathered}
$$

The generating function is $\left\langle p, q_{+}\right\rangle+F\left(p, q_{+}\right)$, with $F$ being a small function. So we have $\psi_{1} \rightarrow I$. At the same time, $\psi_{1}$ is also satisfied with $\left(\widehat{p}_{+}, \widehat{q}_{+}\right) \rightarrow(\widehat{p}, \widehat{q})$ by

$$
\begin{gathered}
\widehat{q}=\widehat{q}_{+}+F_{1}\left(\widehat{p}, \widehat{q}_{+}\right), \\
\widehat{p}_{+}=\widehat{p}+F_{2}\left(\widehat{p}, \widehat{q}_{+}\right) .
\end{gathered}
$$

By (21) to (23), we have a conjugate mapping $\bar{\Phi}=\psi_{1}^{-1}$ 。 $\Phi \circ \psi_{1}:\left(p_{+}, q_{+}\right) \rightarrow\left(\widehat{p}_{+}, \widehat{q}_{+}\right)$implicitly by

$$
\begin{aligned}
& \widehat{p}_{+}=H_{2}(p, \widehat{q})+F_{2}\left(\hat{p}, \widehat{q}_{+}\right), \\
& q_{+}=H_{1}(p, \widehat{q})-F_{1}\left(p, q_{+}\right) .
\end{aligned}
$$

We will prove that there is a function $\bar{H}\left(p_{+}, \widehat{q}_{+}\right)$generating $\bar{\Phi}$.

Lemma 7 (Lemma 2.1 in [18]). The conjugate symplectic mapping $\bar{\Phi}$ can be implicitly determined by a generating function $\bar{H}\left(p_{+}, \widehat{q}_{+}\right)$through

$$
\begin{aligned}
& \widehat{p}_{+}=\partial_{\widehat{q}_{+}} \bar{H}\left(p_{+}, \widehat{q}_{+}\right), \\
& q_{+}=\partial_{p_{+}} \bar{H}\left(p_{+}, \widehat{q}_{+}\right),
\end{aligned}
$$

where

$$
\begin{aligned}
\bar{H}\left(p_{+}, \widehat{q}_{+}\right)= & H(p, \widehat{q})+H_{1}(p, \widehat{q}) F_{2}\left(p, q_{+}\right) \\
& -H_{2}(p, \widehat{q}) F_{1}\left(\widehat{p}, \widehat{q}_{+}\right)+F\left(\widehat{p}, \widehat{q}_{+}\right) \\
& -F\left(p, q_{+}\right)-F_{1}\left(p, q_{+}\right) F_{2}\left(p, q_{+}\right),
\end{aligned}
$$

where $p, \hat{p}, \hat{q}, q_{+}$depend on $\left(p_{+}, \widehat{q}_{+}\right)$as explained above.

Moreover, set $z=\left(p_{+}, \widehat{q}_{+}\right), \Delta z=\left(-F_{2}\left(p, q_{+}\right), F_{1}\left(\hat{p}, \widehat{q}_{+}\right)\right)$, and then we have

$$
\begin{aligned}
\bar{H}(z)= & H(z)+F\left(N_{2}(z), \widehat{q}_{+}\right)-F\left(p_{+}, N_{1}(z)\right) \\
& +Q(z) .
\end{aligned}
$$

The small term $Q(z)$ has the estimate

$$
\left\|X_{\mathrm{Q}}\right\|_{r ; \mathscr{D}(s-5 \rho, r / 16) \times \Pi} \leq \frac{c \epsilon^{2}}{\alpha^{2 \bar{v}} \rho^{2 v}}
$$

with $\bar{v}=4(m+1), v=m+n+4 \tau(m+1)$.
2.1.2. Homological Equations. Now we will solve homological equations for $F$. Let

$$
P=R+\widetilde{P}
$$

where

$$
\begin{aligned}
R(p, \widehat{q})= & P_{000}(x)+\left\langle P_{100}(x), \widehat{y}\right\rangle+\left\langle P_{010}(x), u\right\rangle \\
& +\left\langle P_{001}(x), \widehat{v}\right\rangle+\left\langle P_{011}(x) u, \widehat{v}\right\rangle \\
& +\frac{1}{2}\left\langle P_{020}(x) u, u\right\rangle+\frac{1}{2}\left\langle P_{002}(x) \widehat{v}, \widehat{v}\right\rangle,
\end{aligned}
$$

with

$$
P_{l i j}=\left.\frac{\partial^{l+i+j} P}{\partial \hat{y}^{l} \partial u^{i} \partial \widehat{v}^{j}}\right|_{u=0, \hat{y}=0, \widehat{v}=0,} \quad 2 l+i+j \leq 2 .
$$

Let $F(p, \widehat{q})$ possess the same form as (30). tions:

We will use the idea in [22] to solve homological equa-

$$
\begin{gathered}
N\left(p_{+}, \hat{q}_{+}\right)+R\left(p_{+}, \hat{q}_{+}\right)-F\left(p_{+}, N_{p}\left(p_{+}, \hat{q}_{+}\right)\right) \\
+F\left(N_{q}\left(p_{+}, \widehat{q}_{+}\right), \widehat{q}_{+}\right)=\bar{N}\left(p_{+}, \widehat{q}_{+}\right) .
\end{gathered}
$$

For simplicity, here and below we drop the subscripts “+” in $p_{+}$and $\widehat{q}_{+}$.

Let $x+\omega=\tilde{x}$. Since

$$
\begin{aligned}
\widehat{p} & =N_{\widehat{q}}(p, \widehat{p})=(\widetilde{x}, A u+C v), \\
q & =N_{p, \widehat{q}}(p, \widehat{p})=(\widehat{y}, A \widehat{v}+B u),
\end{aligned}
$$

we have

$$
\begin{aligned}
F & \left(N_{q}\left(p_{+}, \widehat{q}_{+}\right), \hat{q}_{+}\right)-F\left(p_{+}, N_{p}\left(p_{+}, \hat{q}_{+}\right)\right) \\
& =L_{0}+L_{1}+L_{2},
\end{aligned}
$$

where $L_{0}, L_{1}, L_{2}$ indicate the $0,1,2$ order terms of $u, \widehat{v}$, respectively, with

$$
\begin{aligned}
L_{0} & =\left(F_{000}(\tilde{x})-F_{000}(x)\right)+\left\langle F_{100}(\tilde{x})-F_{100}(x), \widehat{y}\right\rangle, \\
L_{1} & =\left\langle A^{T} F_{010}(\tilde{x})-F_{010}(x)-B F_{001}(x), u\right\rangle \\
& +\left\langle C F_{010}(\tilde{x})+F_{001}(\tilde{x})-A F_{001}(x), v\right\rangle, \\
L_{2} & =\left\langle\left\{ F_{011}(\tilde{x}) A-A F_{011}(x)+C F_{020}(\tilde{x}) A\right.\right. \\
& \left.\left.-A F_{002}(x) B\right\} u, \widehat{v}\right\rangle+\frac{1}{2}\left\langle\left\{ A^{T} F_{020}(\tilde{x}) A-F_{020}(x)\right.\right. \\
& \left.\left.-B F_{002}(x) B-B F_{011}(x)-F_{011}^{T}(x) B\right\} u, u\right\rangle \\
& +\frac{1}{2}\left\langle\left\{ C F_{020}(\tilde{x}) C+F_{002}(\tilde{x})-A F_{002}(x) A^{T}\right.\right. \\
& \left.\left.+F_{011}(\tilde{x}) C+C F_{011}^{T}(\tilde{x})\right\} \widehat{v}, \widehat{v}\right\rangle .
\end{aligned}
$$


Let $A=\operatorname{diag}\left(a_{1}, \ldots, a_{n}\right)$ and $B=C=\operatorname{diag}\left(b_{1}, \ldots, b_{n}\right)$ with $a_{j}=\sec \theta_{j}$ and $b_{j}=\tan \theta_{j}$. Now we solve the following equations:

$$
\begin{aligned}
& L_{0}=\left(R_{000}(x)-\left[R_{000}\right]\right)+\left(R_{100}(x)-\left[R_{100}\right]\right) \\
& L_{1}=R_{010}(x)+R_{001}(x) \\
& L_{2}=\left(R_{011}(x)-\widehat{A}\right)+\left(R_{020}-\widehat{B}\right)+\left(R_{002}-\widehat{C}\right),
\end{aligned}
$$

where $\widehat{A}, \widehat{B}$, and $\widehat{C}$ will be determined later.

Firstly, for $F_{j 00}, j=0,1$, we consider the equation

$$
F_{j 00}(x+\omega)-F_{j 00}(x)=R_{j 00}(x)-\left[R_{j 00}\right] .
$$

We now expand the following functions as Fourier series: $F_{j 00}(x)=\sum_{k \in \mathbb{Z}^{n}} F_{k j 00} e^{i\langle k, x\rangle}$ and $R_{j 00}(x)=\sum_{k \in \mathbb{Z}^{n}} R_{k j 00} e^{i\langle k, x\rangle}$. Then we get

$$
F_{k j 00}=\frac{1}{e_{k}-1} R_{k j 00}
$$

with $e_{k}=e^{i\langle k, \omega\rangle}, k \neq 0$.

Next we try to get $F_{010}$ and $F_{001}$. Let $F_{010}=\left(F_{010}^{1}, \ldots, F_{010}^{m}\right)$ and $F_{001}=\left(F_{001}^{1}, \ldots, F_{001}^{m}\right)$. We expand the following functions as Fourier series: $F_{0 i^{\prime} j^{\prime}}^{l}(x)=\sum_{k \in \mathbb{Z}^{n}} F_{k 0 i^{\prime} j^{\prime}}^{l} e^{i\langle k, x\rangle}$ and $R_{0 i^{\prime} j^{\prime}}^{l}(x)=\sum_{k \in \mathbb{Z}^{n}} R_{k 0 i^{\prime} j^{\prime}}^{l} e^{i\langle k, x\rangle}$ with $l=1,2, \ldots, m$ and $\left(i^{\prime}, j^{\prime}\right)=(0,1),(1,0)$.

To get the relations between $F_{0 i^{\prime} j^{\prime}}^{l}(x)$ and $R_{0 i^{\prime} j^{\prime}}^{l}(x)$, we need the following equation set:

$$
\left(\begin{array}{cc}
a e_{k}-1 & -b \\
b e_{k} & e_{k}-a
\end{array}\right) \cdot\left(\begin{array}{c}
F_{k 010}^{l}(x) \\
F_{001}(x)
\end{array}\right) \cdot e^{i\langle k, x\rangle}=\left(\begin{array}{c}
R_{k 010}^{l} \\
R_{k 001}^{l}
\end{array}\right)
$$

with $a=\sec \theta_{l}, b=c=\tan \theta_{l}$. Then we have

$$
F_{k 0 i^{\prime} j^{\prime}}^{l}=\frac{\widetilde{R}_{i^{\prime} j^{\prime}}^{l}}{2 \sec \theta_{j} e_{k}\left(\cos \langle k, \omega\rangle-\cos \theta_{j}\right)},
$$

where $\widetilde{R}_{i^{\prime} j^{\prime}}^{l}$ are linear combination of $R_{010}^{l}(x)$ and $R_{001}^{l}(x)$.

Thirdly, we solve the third equation of (36). For this purpose, we solve

$$
L_{2}=R_{011}(x)+R_{020}+R_{002}
$$

$$
\text { Let } F_{0 i^{\prime} j^{\prime}}=\left(F_{0 i^{\prime} j^{\prime}}^{i j}\right)_{1 \leq i, j \leq m} \text { with }\left(i^{\prime}, j^{\prime}\right)=(1,1),(2,0),(0,2) \text {. }
$$
We expand the following functions: $F_{0 i^{\prime} j^{\prime}}^{i j}=\sum_{k \in \mathbb{Z}^{n}} F_{k 0 i^{\prime} j^{\prime}} e^{i\langle k, x\rangle}$ and $R_{0 i^{\prime} j^{\prime}}^{i j}=\sum_{k \in \mathbb{Z}^{n}} R_{k 0 i^{\prime} j^{\prime}} e^{i\langle k, x\rangle}$.

To get the relations between $F_{k 0 i^{\prime} j^{\prime}}^{i j}$ and $R_{010}^{i j}(x)$, we need the following equation set:

$$
\begin{aligned}
& \left(\begin{array}{cccc}
0 & e_{k} a_{j}-a_{i} & e_{k} b_{i} a_{j} & -a_{i} b_{j} \\
e_{k} a_{i}-a_{j} & 0 & e_{k} a_{i} b_{j} & -b_{i} a_{j} \\
-b_{j} & -b_{i} & e_{k} a_{i} a_{j}-1 & -b_{i} b_{j} \\
e_{k} b_{i} & e_{k} b_{j} & e_{k} b_{i} b_{j} & e_{k}-a_{i} a_{j}
\end{array}\right) \\
& \left(\begin{array}{c}
F_{k 011}^{j i} \\
F_{k 011}^{i j} \\
F_{k 020}^{i j} \\
F_{k 002}^{i j}
\end{array}\right) \cdot e^{i\langle k, x\rangle}=\left(\begin{array}{c}
R_{k 011}^{j i} \\
R_{k 011}^{i j} \\
R_{k 020}^{i j} \\
R_{k 002}^{i j}
\end{array}\right) .
\end{aligned}
$$

Then we have

$$
F_{k 0 i^{\prime} j^{\prime}}^{i j}=\frac{\widetilde{R}^{i j}}{4 \sec \theta_{i} \sec \theta_{j}\left(\cos \langle k, \omega\rangle-\cos \left(\theta_{i}+\theta_{j}\right)\right)\left(\cos \langle k, \omega\rangle-\cos \left(\theta_{i}-\theta_{j}\right)\right)},
$$

with $\widetilde{R}^{i j}$ being linear combination of $R_{k 0 i^{\prime} j^{\prime}}^{i j}$.

2.1.3. Extension of Small Divisors. This part is critical for this paper. Let $\phi(t)$ be a $C^{\infty}(\mathbb{R})$-smooth function with

$$
f(t)= \begin{cases}0, & |t| \leqslant \frac{1}{2} \\ 1, & |t| \geqslant 1\end{cases}
$$

For $h \geqslant 0$, let $f_{h}(t)=f(t / h)$. Then $f_{h}(t) \in C^{\infty}(\mathbb{R})$ with

$$
\left|\frac{d^{\lambda}}{d t^{\lambda}} f_{h}(t)\right| \leqslant \frac{c_{\lambda}}{h^{\lambda}}, \quad \forall t \in \mathbb{R}, \forall \lambda \geqslant 0,
$$

where $c_{\lambda}$ is a constant depending on $\lambda$.
Firstly, we extend $F_{k j 00}$ from $\mathcal{U}$ to the whole set $\Pi$. Let

$$
\begin{aligned}
h & =\frac{\alpha}{(1+|k|)^{\tau}}, \\
t_{1 k}(\xi) & =\langle\omega(\xi), k\rangle-2 \pi l, \\
g_{1 k}(\xi) & =\frac{f_{h}\left(t_{1 k}(\xi)\right)}{e^{i\langle\omega(\xi), k\rangle}-1}, \quad \forall \xi \in \Pi .
\end{aligned}
$$

By the definition of $\mathcal{U}$, we have

$$
g_{1 k}(\xi)=\frac{1}{e^{i\langle\omega(\xi), k\rangle}-1}, \quad \forall \xi \in \mathcal{U}
$$


Note that even if $\mathscr{U}=\emptyset$, the extension of $g_{1 k}(\xi)$ is still well defined on $\Pi$. Furthermore, $g_{1 k}(\xi) \in C^{m}(\Pi)$ with estimate

$$
\left|\frac{\partial^{\beta} g_{1 k}}{\partial \xi^{\beta}}\right| \leqslant \frac{c|k|^{\beta}}{\lambda^{|\beta|+1}}, \quad \xi \in \Pi, \forall|\beta| \leqslant m .
$$

Noticing (38), we now extend $F_{k j 00}$ from $\mathcal{U}$ to whole set $\Pi$ by setting

$$
\begin{array}{r}
\widetilde{F}_{k j 00}(\xi)=g_{1 k}(\xi) R_{k j 00}(\xi)=\frac{f_{q}\left(t_{k}(\xi)\right)}{e^{i\langle\omega(\xi), k\rangle}-1} R_{k j 00}(\xi), \\
j=0,1,0<|k| \leqslant K .
\end{array}
$$

Let

$$
\widetilde{F}_{j 00}(\xi ; x, y)=\sum_{0<|k| \leqslant K} \widetilde{F}_{k j 00}(\xi ; y) e^{i\langle\omega(\xi), k\rangle} .
$$

Then we have the following estimates:

$$
\left\|\widetilde{F}_{j 00}\right\|_{(s-\rho) \times \Pi} \leq \frac{c\left\|R_{j 00}\right\|_{s}}{\alpha^{m+1} \rho^{m+n+\tau(m+1)}} .
$$

Secondly, we extend $F_{k 0 i^{\prime} j^{\prime}}^{l}$ from $\mathcal{U}$ to the whole set $\Pi$, where $\left(i^{\prime}, j^{\prime}\right)=(0,1),(1,0)$. Let

$$
\begin{aligned}
t_{2 k} & =\langle\omega(\xi), k\rangle-\theta-2 \pi l, \\
g_{2 k}(\xi) & =\frac{f_{h}\left(t_{2 k}(\xi)\right)}{2 \sec \theta_{j} e_{k}\left(\cos \langle k, \omega\rangle-\cos \theta_{j}\right)} .
\end{aligned}
$$

Then we have

$$
g_{2 k}(\xi)=\frac{1}{2 \sec \theta_{j} e_{k}\left(\cos \langle k, \omega\rangle-\cos \theta_{j}\right)}, \quad \forall \xi \in \mathcal{U}
$$

Noticing (40), we extend $F_{k 0 i^{\prime} j^{\prime}}^{l}$ by setting

$$
\begin{aligned}
\widetilde{F}_{k 0 i^{\prime} j^{\prime}}^{l}(\xi) & =g_{2 k}(\xi) \widetilde{R}_{i^{\prime} j^{\prime}}^{l} \\
& =\frac{f_{h}\left(t_{k}(\xi)\right)}{2 \sec \theta_{j} e_{k}\left(\cos \langle k, \omega\rangle-\cos \theta_{j}\right)} \widetilde{R}_{i^{\prime} j^{\prime}}^{l},
\end{aligned}
$$

with $l=1,2, \ldots, m$ and $\left(i^{\prime}, j^{\prime}\right)=(0,1),(1,0), 0<|k| \leqslant K$. Let

$$
\widetilde{F}_{0 i^{\prime} j^{\prime}}(\xi ; x, y)=\sum_{0<|k| \leqslant K} \widetilde{F}_{k 0 i^{\prime} j^{\prime}}(\xi ; y) e^{i\langle\omega(\xi), k\rangle} .
$$

Then we have

$$
\left\|\widetilde{F}_{0 i^{\prime} j^{\prime}}\right\|_{D(s-\rho, r) \times \Pi} \leq \frac{c\left\|R_{0 i^{\prime} j^{\prime}}\right\|_{s}}{\alpha^{2 m+2} \rho^{2 \tau(m+1)+m+n}}
$$

with $\left(i^{\prime}, j^{\prime}\right)=(0,1),(1,0)$.

At last, we expend $F_{k 0 i^{\prime} j^{\prime}}^{i j}$, where $1 \leq i, j \leq m$ and $\left(i^{\prime}, j^{\prime}\right)=$ $(1,1),(2,0),(0,2)$. Let

So we have

$$
g_{3 k}(\xi)=\frac{1}{4 \sec \theta_{i} \sec \theta_{j}\left(\cos \langle k, \omega\rangle-\cos \left(\theta_{i}+\theta_{j}\right)\right)\left(\cos \langle k, \omega\rangle-\cos \left(\theta_{i}-\theta_{j}\right)\right)},
$$

$\forall \xi \in \mathcal{U}$. Noting (43), we now extend $F_{k 0 i^{\prime} j^{\prime}}^{i j}$ to the whole set $\Pi$ by setting

$$
\widetilde{F}_{k 0 i^{\prime} j^{\prime}}^{i j}=g_{3 k}(\xi) \widetilde{R}^{i j}, \quad 0<|k|<K .
$$

Let

$$
\widetilde{F}_{0 i^{\prime} j^{\prime}}^{i j}=\sum_{0<|k| \leqslant K} \widetilde{F}_{k 0 i^{\prime} j^{\prime}}^{i j} e^{i\langle\omega(\xi), k\rangle} .
$$

Recalling the third equation of (36) and the conclusions of (41), we let

$$
\begin{aligned}
& \widehat{\omega}=\operatorname{diag}\left(\widehat{\omega}_{1}, \ldots, \widehat{\omega}_{n}\right), \\
& \widehat{A}=\operatorname{diag}\left(\widehat{A}_{1}, \ldots, \widehat{A}_{m}\right), \\
& \widehat{B}=\operatorname{diag}\left(\widehat{B}_{1}, \ldots, \widehat{B}_{m}\right), \\
& \widehat{C}=\operatorname{diag}\left(\widehat{C}_{1}, \ldots, \widehat{C}_{m}\right),
\end{aligned}
$$


with

$$
\begin{aligned}
& \widehat{\omega}_{j}=\left[R_{100}^{j j}\right], \\
& \widehat{A}_{j}=\left[R_{011}^{j j}\right], \\
& \widehat{B}_{j}=\left[R_{020}^{j j}\right], \\
& \widehat{C}_{j}=\left[R_{002}^{j j}\right] .
\end{aligned}
$$

We sum up the above discussion and arrive at

$$
\left\|\widetilde{F}_{0 i^{\prime} j^{\prime}}\right\|_{D(s-\rho, r) \times \Pi} \leq \frac{c\left\|R_{0 i^{\prime} j^{\prime}}\right\|_{s}}{\alpha^{4 m+4} \rho^{4 \tau(m+1)+m+n}}
$$

with $\left(i^{\prime}, j^{\prime}\right)=(1,1),(2,0),(0,2)$.

By the above discussion, we get a function $\widetilde{F}$ defined on $\Pi$ which is the extension of $F$. Moreover $\widetilde{F}$ not only has the same form as $R$ and $F$, but also inherits all properties of $F$ when $\xi \in \mathcal{U}$. So $\widetilde{F}$ satisfies $\left(L_{0}+L_{1}+L_{2}\right) \widetilde{F}=R-\widehat{N}$, where

$$
\begin{aligned}
\widehat{N}(p, \widehat{q})= & \langle\widehat{\omega}, \widehat{y}\rangle+\langle\widehat{A} u, \widehat{v}\rangle+\frac{1}{2}\langle\widehat{B} u, u\rangle \\
& +\frac{1}{2}\langle\widehat{C} \hat{v}, \widehat{v}\rangle .
\end{aligned}
$$

So we have

$$
\left\|X_{\widetilde{F}}\right\|_{r ; D(s-\rho, r) \times \Pi} \leq \frac{c \epsilon}{\alpha^{\bar{v}} \rho^{v}} .
$$

Let $\chi:(p, q) \rightarrow\left(-\widetilde{F}_{y_{+}}, \widetilde{F}_{x}\right)$. Since $\psi_{1}=i d+\chi$, we combine Cauchy estimate to obtain

$$
\begin{gathered}
\left\|\psi_{1}-i d\right\|_{r ; D(s-3 \rho, r / 4) \times \Pi} \leq \frac{c \epsilon}{\alpha^{\bar{v}} \rho^{v}}, \\
\left\|D \psi_{1}-i d\right\|_{r ; D(s-3 \rho, r / 4) \times \Pi} \leq \frac{c \epsilon}{\alpha^{\bar{v}} \rho^{\nu+1}} .
\end{gathered}
$$

2.1.4. Normal Form. From the above discussion, we get a conjugate mapping $\bar{\Phi}(\cdot ; \xi)=\psi_{1}^{-1} \circ \Phi \circ \psi_{1}$ generated by $\bar{H}=\bar{N}_{1}+\bar{N}_{2}+\bar{P}$ where $\bar{N}_{1}=\langle x+\omega+\widehat{\omega}, \hat{y}\rangle, \bar{N}_{2}=$ $\langle\bar{A} u, \widehat{v}\rangle+(1 / 2)\langle\bar{B} u, u\rangle+(1 / 2)\langle\bar{C} \hat{v}, \widehat{v}\rangle$, and $\bar{P}=\widetilde{P}+Q$ with

$$
\begin{aligned}
& \widehat{\omega}=\omega_{+}-\omega, \\
& \bar{A}=A_{0}+\widehat{A}, \\
& \bar{B}=B_{0}+\widehat{B}, \\
& \bar{C}=C_{0}+\widehat{C} .
\end{aligned}
$$

We note that the normal form depends only on $u$, $v$, so we consider $\bar{N}_{2}$. Since $B=C$ and $A^{2}=B^{2}+C^{2}$ may not be satisfied, $\bar{N}_{2}$ may not be a normal form and hence we need normalize $\bar{N}_{2}$ into a new normal form.

Lemma 8. There exists a symplectic mapping $\psi_{2}$ generated by $\langle u, \widehat{v}\rangle+G\left(u, v_{+}\right)$, such that the corresponding conjugate mapping $\Phi_{+}(\cdot ; \xi)=\bar{\psi}_{2}^{-1} \circ \bar{\Phi} \circ \bar{\psi}_{2}:\left(u_{+}, v_{+}\right) \rightarrow\left(\widehat{u}_{+}, \widehat{v}_{+}\right)$is generated by

$$
\widetilde{N}_{2+}=\left\langle A_{+} u, \widehat{v}\right\rangle+\frac{1}{2}\left\langle B_{+} u, u\right\rangle+\frac{1}{2}\left\langle C_{+} \widehat{v}, \widehat{v}\right\rangle,
$$

where $A_{+}^{2}=B_{+}^{2}+1, B_{+}=C_{+}$. Let $A_{+}=\sec \theta_{+}$. One has

$$
\left\|\theta_{+}-\theta\right\|_{\mathscr{C}^{m}(\Pi)}<c \epsilon
$$

and hence

$$
\bar{\psi}_{2}-i d=O(\epsilon) .
$$

Remark 9. We note that $\bar{\psi}_{2}$ can normalize $\bar{N}_{2}$ into a new normal form. Using the same idea and method, we can find a new symplectic mapping $\psi_{2}$ normalizing $\bar{N}_{1}(x, \widehat{y})+\bar{N}_{2}(u, \widehat{v})$ into

$$
\begin{aligned}
N_{+}= & \left\langle x+\omega_{+}, \hat{y}\right\rangle+\left\langle A_{+} u, \widehat{v}\right\rangle+\frac{1}{2}\left\langle B_{+} u, u\right\rangle \\
& +\frac{1}{2}\left\langle C_{+} \hat{v}, \widehat{v}\right\rangle .
\end{aligned}
$$

In fact, $\psi_{2}=\langle p, \hat{q}\rangle+G\left(p, q_{+}\right)$with $\psi_{2}-i d=O(\epsilon)$. have

Let $\Psi=\psi_{1} \circ \psi_{2}$. Similar to the estimates of $\psi_{1}$ and $\psi_{2}$, we

$$
\begin{gathered}
\|\Psi-i d\|_{r ; \mathscr{D}(s-3 \rho, r / 4) \times \Pi} \leq \frac{c \epsilon}{\alpha^{\bar{\nu}} \rho^{\nu}}, \\
\|D \Psi-I d\|_{r ; \mathscr{D}(s-3 \rho, r / 4) \times \Pi} \leq \frac{c \epsilon}{\alpha^{\bar{\nu}} \rho^{\nu+1}} .
\end{gathered}
$$

Moreover, $H_{+}=N_{+}+P_{+}$, where $N_{+}$is normal form. $H_{+}$can be shown just like $\bar{H}$, and we omit the details.

2.1.5. Estimates for New Perturbation and Error Terms. We aim at the estimate of $P_{+}$and some error terms. By (29), we have

$$
\left\|X_{\tilde{P}}\right\|_{\eta r ; \mathscr{D}(s-5 \rho, \eta r) \times \Pi} \leq c \cdot \epsilon\left(\eta+\frac{e^{-K \rho}}{\eta^{2}}\right) .
$$

By (28) and (74), we have

$$
\left\|X_{P_{+}}\right\|_{\eta r ; \mathscr{D}(s-5 \rho, \eta r) \times \Pi} \leq c \cdot \epsilon\left(\eta+\frac{e^{-K \rho}}{\eta^{2}}\right)+\frac{c \epsilon^{2}}{\eta^{2} \alpha^{2 \bar{v}} \rho^{2 \nu}} .
$$

Moreover, for the error terms $\widehat{\omega}=\omega_{+}-\omega$ and $\widehat{\theta}=\theta_{+}-\theta$, we have $\|\widehat{\omega}\|_{\mathscr{C}^{m}(\Pi)} \leq \epsilon$ and $\|\widehat{\theta}\|_{\mathscr{C}^{m}(\Pi)} \leq c \epsilon$.

2.1.6. The Choice of Parameters in KAM Iteration Lemma. We choose a weighted error $0<E<1$ and set

$$
\begin{aligned}
& \eta=E, \\
& \epsilon=\alpha^{2 \bar{v}} \rho^{2 v} E \eta^{2}, \\
& E=\frac{e^{-K \rho}}{\eta^{2}}, \\
& h=\frac{\alpha}{2 K^{\tau+1} T} .
\end{aligned}
$$


Fix $\sigma \in(0,1)$, for the next step, we define

$$
\begin{aligned}
& \rho_{+}=\sigma \rho, \\
& s_{+}=s-5 \rho, \\
& r_{+}=\eta r, \\
& \alpha_{+}=\alpha+3(1+K)^{\tau+1} \epsilon, \\
& \epsilon_{+}=c \eta \epsilon, \\
& E_{+}=c E^{4 / 3} .
\end{aligned}
$$

By (75) and $\alpha<2 \alpha_{+}$, we have

$$
\begin{aligned}
\left\|X_{P_{+}}\right\|_{\eta r ; \mathscr{D}(s-5 \rho, \eta r) \times \Pi} & \leq c \cdot \epsilon\left(\eta+\frac{e^{-K \rho}}{\eta^{2}}\right)+\frac{c \epsilon^{2}}{\eta^{2} \alpha^{2 \bar{v}} \rho^{2 v}} \\
& \leq c \eta \epsilon=c \alpha^{2 \bar{v}} \rho^{2 v} E^{4} \leq c \alpha_{+}^{2 \bar{v}} \rho_{+}^{2 v} E_{+}^{3} .
\end{aligned}
$$

Setting $\epsilon_{+}=c \alpha_{+}^{2 \bar{v}} \rho_{+}^{2 v} E_{+}^{3}$, we arrive at

$$
\left\|X_{P_{+}}\right\|_{r_{+} ; \mathscr{D}\left(s_{+}, r_{+}\right) \times \Pi} \leq \epsilon_{+} .
$$

At last, let $\alpha_{+}=\alpha_{+}=\alpha+3(1+K)^{\tau+1} \epsilon$, and then we have $\mathcal{U}_{+} \subset \mathcal{U}$.

2.2. Iteration. In this section, we will summarize the above results on parameters so that KAM-step can iterate infinitely. At the initial step set

$$
\begin{aligned}
& s_{0}=s, \\
& \rho_{0}=\frac{(1-\sigma) s}{10}, \\
& r_{0}=r, \\
& \alpha_{0}=\frac{\alpha}{2}, \\
& E_{0}=E, \\
& \eta_{0}=E_{0}, \\
& \epsilon_{0}=\alpha_{0}^{2 \bar{\nu}} \rho_{0}^{2 v} E_{0} \eta_{0}^{2} .
\end{aligned}
$$

Let

$$
\begin{aligned}
& \frac{e^{-K_{0} \rho_{0}}}{\eta_{0}^{2}}=E_{0}, \\
& \omega_{0}(\xi)=\omega(\xi) .
\end{aligned}
$$

Assume that $\rho_{j}, s_{j}, r_{j}, E_{j}, \alpha_{j}$ are well defined for the $j$ th step. Then $\eta_{j}, K_{j}, \epsilon_{j}$ are defined as follows:

$$
\begin{aligned}
\eta_{j} & =E_{j}, \\
\epsilon_{j} & =\alpha_{j}^{2 \bar{v}} \rho_{j}^{2 v} E_{j} \eta_{j}^{2}, \\
\frac{e^{-K_{j} \rho_{j}}}{\eta_{j}^{2}} & =E_{j} .
\end{aligned}
$$

Define inductive sequences:

$$
\begin{aligned}
\rho_{j+1} & =\sigma \rho_{j}, \\
s_{j+1} & =s_{j}-5 \rho, \\
r_{j+1} & =\eta_{j} r_{j}, \\
\alpha_{j+1} & =\alpha_{j}+3(1+K)_{j}^{\tau+1} \epsilon_{j}, \\
E_{j+1} & =c E_{j}^{4 / 3} .
\end{aligned}
$$

Similarly, we can define $\eta_{j+1}, K_{j+1}, \epsilon_{j+1}$.

Similar to the proof of KAM iteration Lemma, we have $\mathcal{U}_{j+1} \subset \mathcal{U}_{j}$. Moreover, there exists a sequence of $\left\{\Psi_{j}\right\}=\left\{\psi_{1 j}\right.$ 。 $\left.\psi_{2 j}\right\}$, where $\psi_{1 j}$ is generated by $\left\langle p, q_{+}\right\rangle+F_{j}\left(p, q_{+}\right)$and $\psi_{2 j}$ is generated by $\left\langle p, q_{+}\right\rangle+G_{j}\left(p, q_{+}\right)$. Moreover, we have

$$
\left\|\Psi_{j}-i d\right\|_{r_{j} ; \mathscr{D}(s-3 \rho, r / 4) \times \Pi} \leq \frac{c \epsilon}{\alpha^{\bar{v}} \rho^{v}}
$$

$$
\left\|D \Psi_{j}-I d\right\|_{r_{j} ; \mathscr{D}(s-3 \rho, r / 4) \times \Pi} \leq \frac{c \epsilon}{\alpha^{\bar{v}} \rho^{\nu+1}} .
$$

There exists a sequence of symplectic mappings $\left\{\Phi_{j}\right\}$ which are well defined on $\mathscr{D}\left(s_{j}, r_{j}\right)$ and satisfy

$$
\Phi_{j+1}=\left(\Psi^{j}\right)^{-1} \circ \Phi_{j} \circ \Psi^{j}
$$

where $\Psi^{j}=\Psi_{1} \circ \Psi_{2} \circ \cdots \circ \Psi_{j}$. The symplectic mappings $\left\{\Phi_{j}\right\}$ are generated by $H_{j}=N_{j}+P_{j}$, where

$$
\begin{aligned}
N_{j}= & \left\langle x+\omega_{j}(\xi)\right\rangle+\left\langle A_{j} u, \widehat{v}\right\rangle+\frac{1}{2}\left\langle B_{j} u, u\right\rangle \\
& +\frac{1}{2}\left\langle C_{j} \hat{v}, \widehat{v}\right\rangle
\end{aligned}
$$

with $A_{j}=\sec \theta_{j}, B_{j}=C_{j}=\tan \theta_{j}$. Moreover we have

$$
\begin{gathered}
\left\|\omega_{j+1}-\omega_{j}\right\|_{\mathscr{C}^{m}(\Pi)} \leq c \epsilon_{j}, \\
\left\|\theta_{j+1}-\theta_{j}\right\|_{\mathscr{C}^{m}(\Pi)} \leq c \epsilon_{j}
\end{gathered}
$$

$$
\forall j \geq 1 \text {. }
$$

2.3. Convergence of the Iteration. Now we prove the convergence of KAM iteration. In the same way as [22, 23], we have

$$
\begin{aligned}
\left\|\Psi^{j}-\Psi^{j-1}\right\|_{r_{j} ; D\left(s_{j}, r_{j}\right) \times \Pi} & \leq c \alpha_{j-1}^{2 \bar{\nu}} \rho_{j-1}^{2 v} E_{j-1}^{3}, \\
\left\|D\left(\Psi^{j}-\Psi^{j-1}\right)\right\|_{r_{j} ; D\left(s_{j}, r_{j}\right) \times \Pi} & \leq \alpha_{j-1}^{2 \bar{v}} \rho_{j-1}^{2 v-1} E_{j-1}^{3} .
\end{aligned}
$$

Note that $s_{j} \rightarrow s / 2$ and $r_{j} \rightarrow 0$ as $j \rightarrow \infty$. Let $\Psi_{*}=$ $\lim _{j \rightarrow \infty} \Psi_{j}$. Since $\Psi_{j}$ is affine in $y$, we have $\Psi_{j}$ converging to $\Psi_{*}$ on $D(r / 2, s / 2)$ with

$$
\left\|\Psi_{*}-i d\right\|_{r / 2 ; D(s / 2, r / 2) \times \Pi} \leq c(\alpha, \rho, \sigma) E_{0}^{3},
$$

$\forall \beta \in Z_{n}^{+}$ 
Let $P_{*}=\lim _{j \rightarrow \infty} P_{j}$ and $\omega_{*}=\lim _{j \rightarrow \infty} \omega_{j}$. Then $P_{*}$ is real analytic in $(x, y)$ on $D(r / 2, s / 2)$ and $\mathscr{C}^{m}$ in $\xi$ on $\Pi$. Note that $\omega_{j}=\omega+\sum_{i=0}^{j-1} \widehat{\omega}_{j}$. Then we have

$$
\left\|\omega_{*}-\omega_{j}\right\|_{\mathscr{C}^{m}(\Pi)} \leqslant \sum_{i=j}^{\infty} \epsilon_{j} \leqslant 2 \epsilon_{j} .
$$

Particularly,

$$
\left\|\omega_{*}-\omega\right\|_{\mathscr{C}^{m}(\Pi)} \leqslant 2 \epsilon .
$$

Correspondingly, let $H_{j}=N_{j}+P_{j} \rightarrow H_{*}=N_{*}+P_{*}$ satisfy

$$
\begin{aligned}
N_{*}= & \left\langle x+\omega_{*}(\xi), \hat{y}\right\rangle+\left\langle A_{*}(\xi) u, \widehat{v}\right\rangle \\
& +\frac{1}{2}\left\langle B_{*}(\xi) u, u\right\rangle+\frac{1}{2}\left\langle C_{*}(\xi) \widehat{v}, \widehat{v}\right\rangle,
\end{aligned}
$$

where $A_{*}=\sec \theta_{*}$ and $B_{*}=C_{*}=\tan \theta_{*}$ with estimates

$$
\left\|\theta_{*}-\theta\right\|_{\mathscr{C}^{m}(\Pi)} \leq c \epsilon .
$$

Moreover, $P_{*}(p, \widehat{q})=\sum_{2|l|+|i|+|j| \geq 3} P_{l i j}(x) \hat{y}^{l} u^{i} \widehat{v}^{j}$.

Let

$$
\Pi_{*}=\left\{\xi \in \Pi:\left(\omega_{*}(\xi), \theta_{*}(\xi)\right) \in O_{\alpha, \tau}\right\} .
$$

In the sequel we will prove $\Pi_{*} \subset \mathcal{U}_{j}$ for all $j \geqslant 0$, which is equal to proving $\alpha \geqslant \alpha_{j}+4 \epsilon_{j} K_{j}^{\tau+1}$. By the choice of parameters, we have $K_{j+1}^{\tau+1} \epsilon_{j+1} / K_{j}^{\tau+1} \epsilon_{j} \leqslant c E_{j}$ and $K_{0}=$ $-3 \ln E_{0} / \rho_{0}$, so

$$
K_{j}^{\tau+1} \epsilon_{j} \leqslant E_{0}^{\mathcal{c}_{0}} K_{0}^{\tau+1} \epsilon_{0} \leqslant c E_{0}^{\mathcal{c}_{0}+3}\left(\frac{\ln E_{0}}{\rho_{0}}\right)^{\tau+1} \alpha \rho_{0}^{\tau^{\prime}} .
$$

Let $E_{0}$ be small enough such that $8 K_{j}^{\tau+1} \epsilon_{j} \leqslant \alpha / 2$, and then we have

$$
\alpha_{j}+4 \epsilon_{j} K_{j}^{\tau+1}=\frac{\alpha}{2}+4 \sum_{l=1}^{j} K_{l}^{\tau+1} \epsilon_{l} \leqslant \frac{\alpha}{2}+8 K_{j}^{\tau+1} \epsilon_{j} \leqslant \alpha .
$$

So $\Pi_{*} \subset \mathscr{U}_{j}$ and hence $\Pi_{*} \subset \bigcap_{j \geqslant 0} \mathscr{U}_{j}$, for all $j \geqslant 0$. This completes the proof of Theorem 4 .

\section{Competing Interests}

The author declares that he has no competing interests.

\section{Acknowledgments}

The paper was completed during the author's visit to Department of Mathematics of Pennsylvania State University, supported by Nanjing Tech University. The author thanks Professor Mark Levi for his inviting, hospitality, and valuable discussions. The work is supported by Natural Science Foundation of Jiangsu Higher Education Institutions of China (14KJB110009).

\section{References}

[1] A. N. Kolmogorov, "On conservation of conditionally periodic motions for a small change in Hamilton's function," Doklady Akademii Nauk SSSR, vol. 98, pp. 527-530, 1954.

[2] A. D. Bruno, "Analytic form of differential equations," Trudy Moskovskogo Matematicheskogo Obshchestva, vol. 26, pp. 199239, 1972.

[3] H. Rüssmann, On twist Hamiltonian, Talk on the Colloque International, Mécanique Céleste et Systèmes Hamiltoniens, Marseille, France, 1990

[4] H. Rüssmann, "Invariant tori in non-degenerate nearly integrable Hamiltonian systems," Regular \& Chaotic Dynamics. International Scientific Journal, vol. 6, no. 2, pp. 119-204, 2001.

[5] J. Xu, J. You, and Q. Qiu, "Invariant tori for nearly integrable Hamiltonian systems with degeneracy," Mathematische Zeitschrift, vol. 226, no. 3, pp. 375-387, 1997.

[6] V. Arnold, "Proof of A.N. Kolmogorov's theorem on conservation of conditionally periodic motions under small perturbations of the hamiltonian function," Uspekhi Matematicheskii Nauk, no. 18, pp. 13-40, 1963.

[7] J. Moser, On Invariant Curves of Area-Preserving Mappings of an Annulus, Vandenhoeck \& Ruprecht, 1962.

[8] J. Moser, "Convergent series expansions for quasi-periodic motions," Mathematische Annalen, vol. 169, pp. 136-176, 1967.

[9] J. Xu and X. Lu, "General KAM theorems and their applications to invariant tori with prescribed frequencies," Regular and Chaotic Dynamics, vol. 21, no. 1, pp. 107-125, 2016.

[10] C. Q. Cheng and Y. S. Sun, "Existence of invariant tori in threedimensional measure-preserving mappings," Celestial Mechanics \& Dynamical Astronomy, vol. 47, no. 3, pp. 275-292, 1989.

[11] R. de la Llave and J. D. Mireles James, "Parameterization of invariant manifolds by reducibility for volume preserving and symplectic maps," Discrete and Continuous Dynamical Systems. Series A, vol. 32, no. 12, pp. 4321-4360, 2012.

[12] H. R. Dullin and J. D. Meiss, "Resonances and twist in volumepreserving mappings," SIAM Journal on Applied Dynamical Systems, vol. 11, no. 1, pp. 319-349, 2012.

[13] A. M. Fox and J. D. Meiss, "Greene's residue criterion for the breakup of invariant tori of volume-preserving maps," Physica D. Nonlinear Phenomena, vol. 243, pp. 45-63, 2013.

[14] V. Gelfreich, C. Simó, and A. Vieiro, "Dynamics of 4D symplectic maps near a double resonance," Physica D. Nonlinear Phenomena, vol. 243, no. 1, pp. 92-110, 2013.

[15] H. Rüssmann, "On the existence of invariant curves of twist mappings of an annulus," in Geometric Dynamics, vol. 1007 of Lecture Notes in Mathematics, pp. 677-718, Springer, Berlin, Germany, 1983.

[16] H. Rüssmann, "Stability of elliptic fixed points of analytic area-preserving mappings under the Bruno condition," Ergodic Theory and Dynamical Systems, vol. 22, no. 5, pp. 1551-1573, 2002.

[17] Z. Xia, "Existence of invariant tori in volume-preserving diffeomorphisms," Ergodic Theory and Dynamical Systems, vol. 12, no. 3, pp. 621-631, 1992.

[18] X. Lu, J. Li, and J. Xu, "A KAM theorem for a class of nearly integrable symplectic mappings," Journal of Dynamics and Differential Equations, pp. 1-24, 2015.

[19] Q. Bi and J. Xu, "Persistence of lower dimensional hyperbolic invariant tori for nearly integrable symplectic mappings," Qualitative Theory of Dynamical Systems, vol. 13, no. 2, pp. 269-288, 2014. 
[20] Z.-j. Shang, "A note on the KAM theorem for symplectic mappings," Journal of Dynamics and Differential Equations, vol. 12, no. 2, pp. 357-383, 2000.

[21] W. Zhu, B. Liu, and Z. Liu, "The hyperbolic invariant tori of symplectic mappings," Nonlinear Analysis: Theory, Methods \& Applications, vol. 68, no. 1, pp. 109-126, 2008.

[22] J. Pöschel, "On elliptic lower dimensional tori in hamiltonian systems," Mathematische Zeitschrift, vol. 202, no. 4, pp. 559-608, 1989.

[23] J. Pöschel, "A lecture on the classical KAM theorem, Smooth ergodic theory and its applications," in Proceedings of the Symposia in Pure Mathematics, vol. 69, pp. 707-732, Seattle, Wash, USA, 1999. 


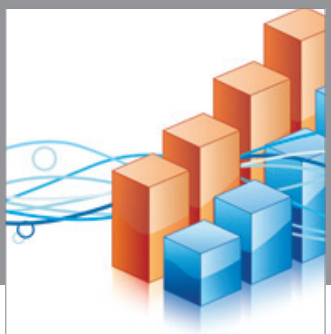

Advances in

Operations Research

vatem alat4

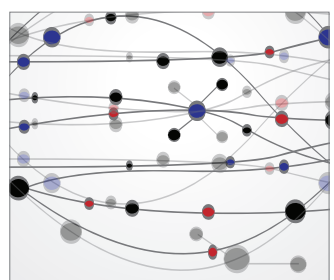

\section{The Scientific} World Journal
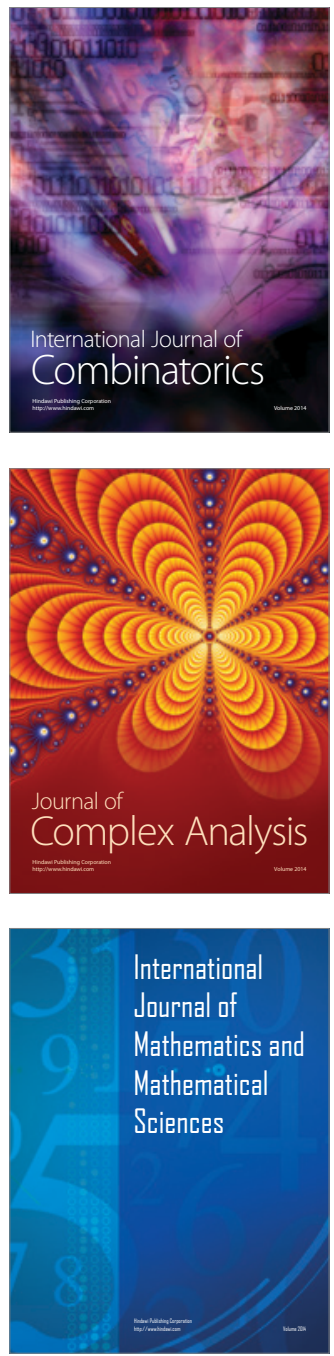
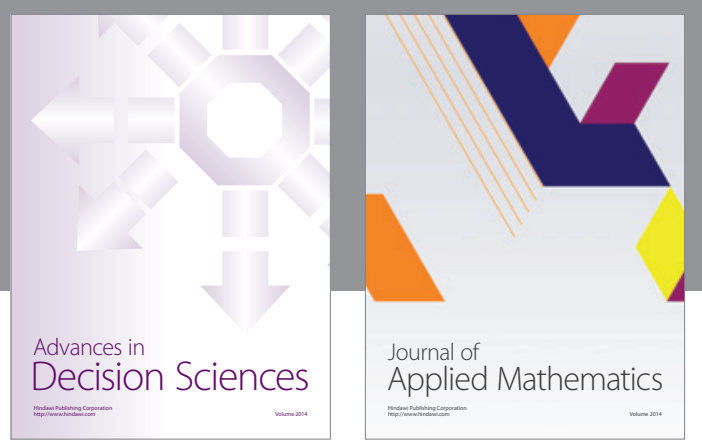

Algebra

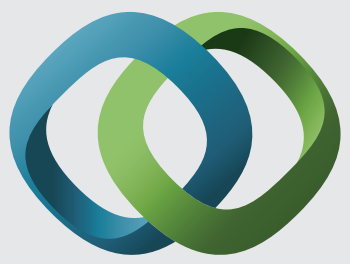

\section{Hindawi}

Submit your manuscripts at

https://www.hindawi.com
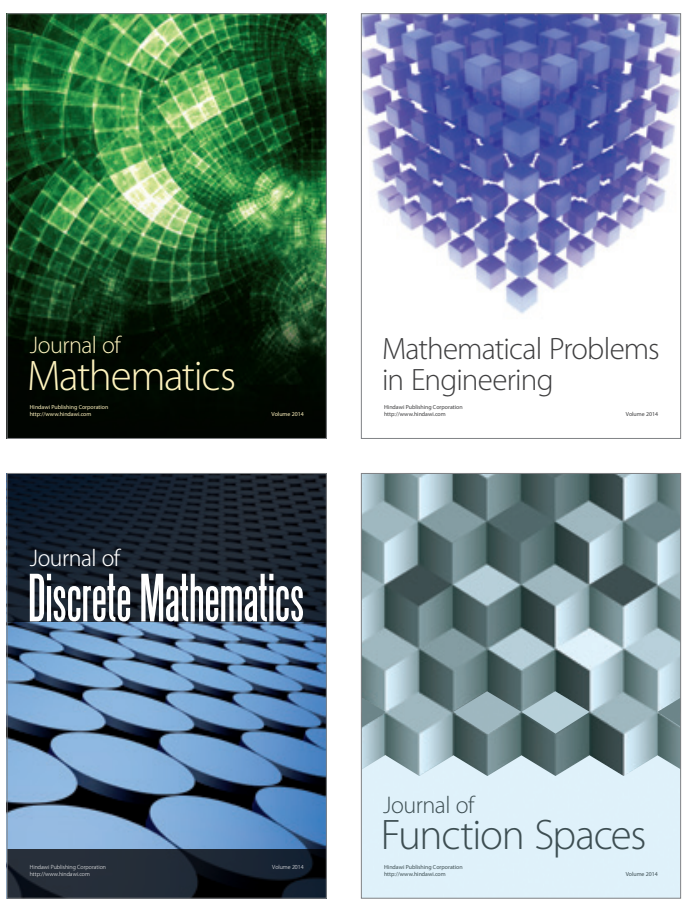

Mathematical Problems in Engineering
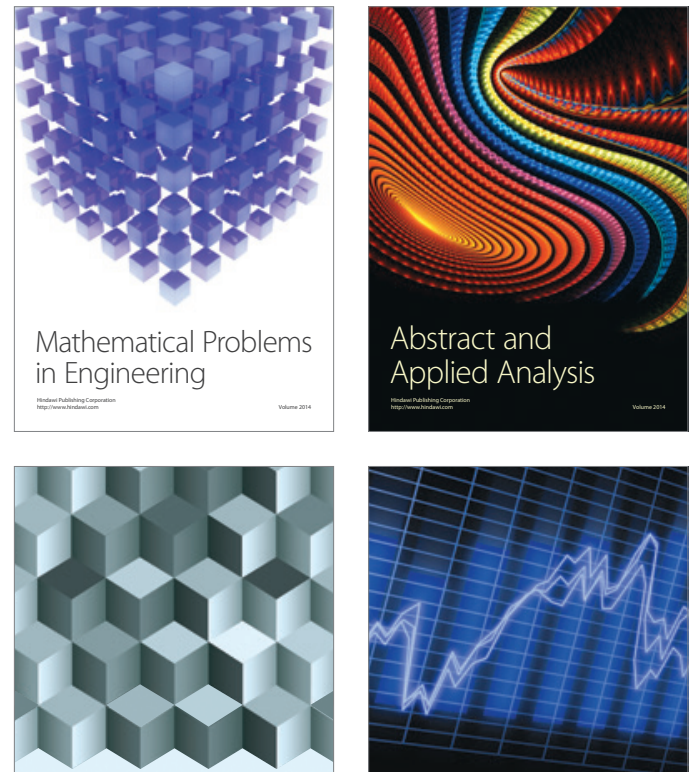

Journal of

Function Spaces

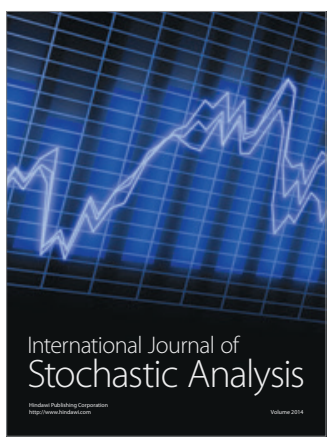

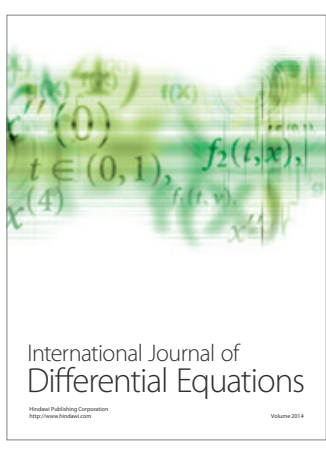
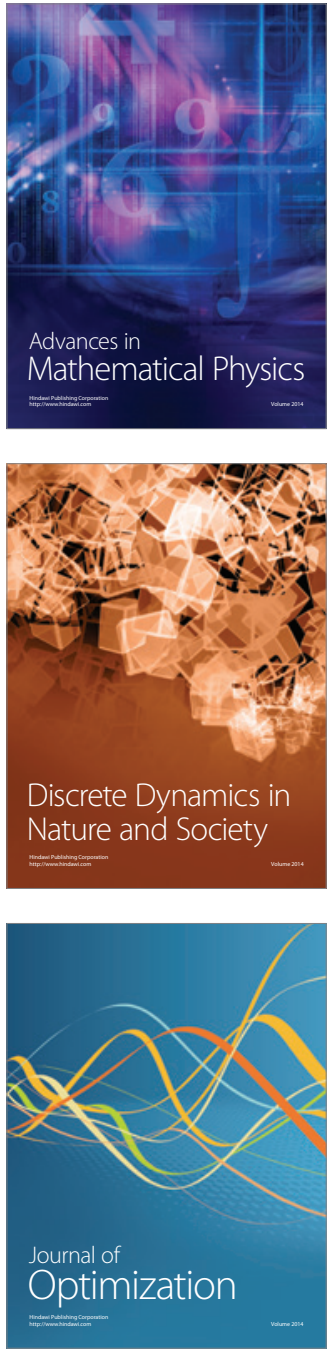